\title{
DJ-1 and its emerging role as a biomarker of systemic malignancies besides lung carcinomas
}

\author{
Shailendra Kapoor
}

Received: 4 May 2012/Accepted: 9 October 2012/Published online: 16 October 2012

(C) Springer Science+Business Media Dordrecht 2012

To the Editor,

I read with great interest the recent article by Bai et al. [1] in a recent issue of your esteemed journal. The article is highly thought provoking. Interestingly, the past few years have seen the emergence of DJ-1 as a significant biomarker of a number of other systemic malignancies besides lung carcinomas.

For instance, marked variation is seen in serum DJ-1 levels in patients with benign prostatic hypertrophy in comparison to serum DJ-1 levels in patients with prostate carcinomas, with higher levels being seen in the latter [2]. As a result, serum DJ-1 appears to be a biomarker with considerable promise for identifying prostate malignancies. Similarly, DJ-1 is emerging as a promising marker of pancreatic carcinomas [3, 4].

Similarly, higher DJ-1 levels have been noted in nipple secretions from breast carcinoma patients and thus DJ-1 serves as a marker of breast malignancy. In fact, Oda et al. [5] have recently reported that a DJ-1 level greater than $3 \mathrm{~nm} / \mathrm{mm}$ has a sensitivity of $75 \%$ in predicting breast carcinomas. Similarly, DJ-1 serves as a biomarker of malignancy in laryngeal squamous cell carcinomas. This is confirmed by the fact that the tumor recurrence rate in laryngeal carcinomas that demonstrate up regulated DJ-1 levels is almost twice that in laryngeal carcinomas that exhibit lower DJ-1 levels [6].

DJ-1 is also emerging as a marker of malignancy in other gastrointestinal malignancies besides pancreatic carcinomas. For instance, hepato-cellular carcinomas exhibit augmented DJ-1 levels in comparison to benign liver

S. Kapoor $(\bowtie)$

2400 Crossing Place, Mechanicsville, VA 23121, USA

e-mail: shailendrakapoor@yahoo.com tissue. Higher DJ-1 levels in fact indicate poor prognosis in hepato-cellular carcinomas [7]. Similarly, DJ-1 promotes tumor proliferation in esophageal carcinomas via the phosphatidylinositol 3-kinase pathway and thereby serves as a marker of poor prognosis in these tumors [8].

The above examples clearly illustrate the significant role of DJ-1 in systemic carcinogenesis and the need for further large scale studies to fully elaborate and understand its carcinogenic effects.

\section{References}

1. Bai J, Guo C, Sun W et al (2012) DJ-1 may contribute to metastasis of non-small cell lung cancer. Mol Biol Rep 39: $2697-2703$

2. Lisitskaia KV, Eremina LS, Ivanov AV et al (2011) Study of Dj-1 protein in tissue specimens, cultured cells and serum of prostate cancer patients. Biomed Khim 57:392-401

3. Chen Y, Kang M, Lu W et al (2012) DJ-1, a novel biomarker and a selected target gene for overcoming chemoresistance in pancreatic cancer. J Cancer Res Clin Oncol 138(9):1463-1474

4. Zeng HZ, Qu YQ, Zhang WJ, Xiu B, Deng AM, Liang AB (2011) Proteomic analysis identified DJ-1 as a cisplatin resistant marker in non-small cell lung cancer. Int J Mol Sci 12:3489-3499

5. Oda M, Makita M, Iwaya K et al (2012) High levels of DJ-1 protein in nipple fluid of patients with breast cancer. Cancer Sci 103(6):1172-1176

6. Zhu XL, Wen WP, Lei WB et al (2010) DJ-1 expression in laryngeal squamous cell carcinoma and its relationship with tumor recurrence and metastasis. Zhonghua Er Bi Yan Hou Tou Jing Wai Ke Za Zhi 45:497-501

7. Liu S, Yang Z, Wei $\mathrm{H}$ et al (2010) Increased DJ-1 and its prognostic significance in hepatocellular carcinoma. Hepatogastroenterology 57:1247-1256

8. Yuen HF, Chan YP, Law S et al (2008) DJ-1 could predict worse prognosis in esophageal squamous cell carcinoma. Cancer Epidemiol Biomarkers Prev 17:3593-3602 